\title{
You Can Wear It, But Do They Want to Share It or Stare at It?
}

\author{
Arto Puikkonen $^{1}$, Anu Lehtiö ${ }^{2}$, and Antti Virolainen ${ }^{3}$ \\ ${ }^{1}$ Nokia Research Center, Visiokatu 1, 33720, Tampere, Finland \\ ${ }^{2}$ Helsinki Institute for Information Technology HIIT, PO Box 68, 00014, \\ University of Helsinki, Finland \\ ${ }^{3}$ Nokia Research Center, Itämerenkatu 11-13, 00180, Helsinki, Finland \\ \{arto.puikkonen, antti.virolainen\}@nokia.com \\ \{anu.lehtio\}@helsinki.fi
}

\begin{abstract}
Wearable technologies are often used for supporting our daily lives instead of aiming to be entertaining. Yet it is in our daily lives that clothing is used to highlight our personas and engage others. In this paper, we describe what type of social acceptance issues might be worth to consider when it comes to entertaining and engaging wearable technology. Our user study with 10 participants was conducted by wearing a T-shirt that served as a display for an online game. The participants wore the T-shirt in their everyday surroundings. We gained a preliminary understanding on peoples' reactions and the suitability of this type of wearable technology for everyday usage. Our results indicate that established social boundaries for inappropriate attention influence the spectator experience with performative wearable technologies.
\end{abstract}

Keywords: Performative Wearable Devices, Social Interaction, Game Spectatorship.

\section{Introduction}

Rapid and continuous miniaturization of electronics and the fast evolution of new materials (e.g. nano materials) bring interesting possibilities for the use of wearable technologies. But despite that fact that wearable solutions prior have mainly dwelled in designs that aid us in our lives, the purchase of clothing is most often based on other factors than the level of aid received. Human actions are performative by nature and we are performing to the people surrounding us, to the spectators of everyday life [1]. Similarly, we choose the clothes we wear based on our own preferences, but also to either avoid or to catch the eye of our spectators. Wearable technologies are an interesting means to provide more elaborate and lively ways to support these needs.

We wanted to test a wearable concept that would support the wearers' desires of expressing self, but would also be entertaining for the spectators and maybe even entice social interaction. For the purpose, we designed a piece of clothing that was visually appealing, appeared to be interactive to enhance the spectator interest towards it and had a social component in the form a game. Our design was aimed to be 
contrary to many other wearable technologies. We aimed heavily on the matter of visual appeal, instead of technological advances.

We chose to design a t-shirt that portrays an on-going game of Tic-Tac-Toe. The shirt was equipped with nine LED displays including a controller unit to control the displays. The purpose was to build the prototype in a way that the person wearing the shirt would not have to worry about the technology but wear it as any normal shirt. For the spectators the form of the T-shirt was supposed to look like a normal T-shirt.

To prevent the shirt becoming rigid and uncomfortable to wear, custom led displays were made. Discrete LEDs were assembled on a flexible substrate instead of using traditional rigid printed wiring boards. Wiring between the displays and a microcontroller was done with conductive strings. A normal T-shirt was used to hide the technology and give a nice finished look and feel while keeping the displays and wirings safe and sound between the two layers of textile (Fig. 1). Pre-programmed tictac-toe game software was stored on the memory of the micro controller and games were run continuously during the test period.

We formed our main research question based on the notion of spectator behavior: Does bringing this type of wearable technology into the context of peoples' everyday environment somehow overstep the social boundaries established for inappropriate attention? To this end we dug deeper into the subjective cognizance of the participants: How did they feel about wearing this type of wearable technology in their everyday environment and their descriptions and interpretations of the reactions and responses of people they came across while wearing the shirt.

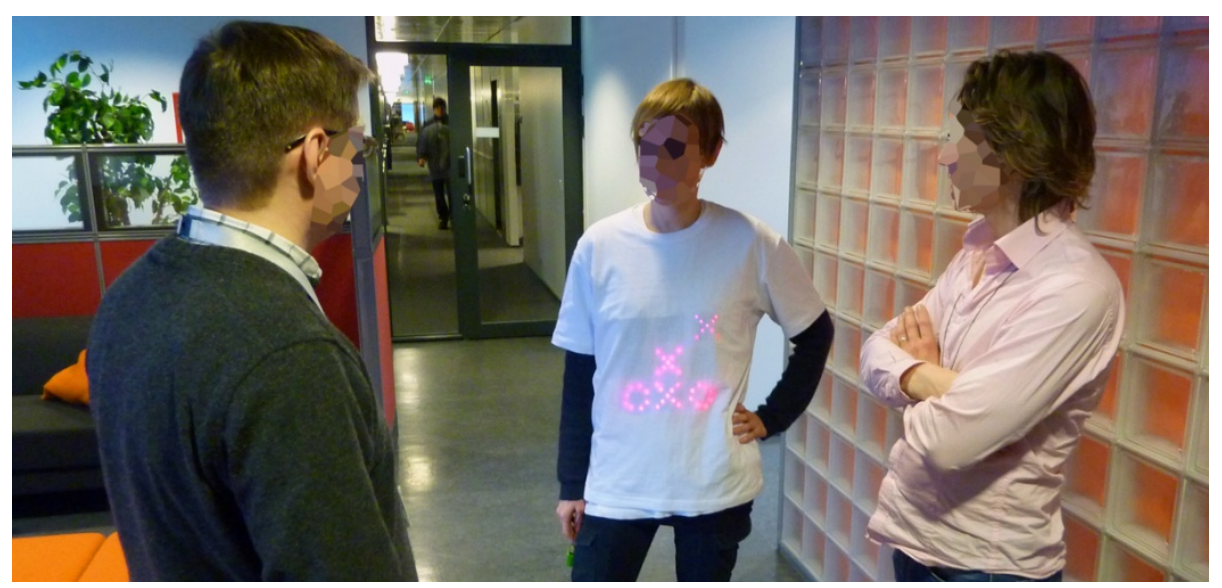

Fig. 1. Tic-Tac-Toe T-shirt in use displaying an on-going game

\section{Related Work}

The key concept contributing to our study from social sciences was 'civil inattention' introduced by Erving Goffman [2]. According to Goffman, social interaction is based on performative actions that take on dramaturgical forms. Bearing that in mind, civil inattention refers to a certain type of behavior that aims at maintaining some privacy 
for the performer in surroundings that are otherwise public. This behavior consists of e.g. showing disinterest, appearing to be absent-minded and/ or acting oblivious as to what's happening. This type of behavior can typically be seen e.g. in urban setting where a lot of people inhabit a limited space. You politely "pretend" not to or make sure that you don't pay too much attention to what others are doing, saying etc.

In the context of games civil inattention is often not beneficial. Quite the opposite, immediate interaction around gaming forms a substantial part of the total experience. When features for social interaction around games are enabled, playing games is more fun and engaging [3,4]. Additionally, Game cafes have been reported to garner their success from supporting conversation around games [5]. Friendships are built and maintained around gaming.

But interaction with the ones around you is not just about socializing. Reeves et al [6] have presented a taxonomy and resulting design principles for designing for the spectator. According to Reeves, interfaces should be approached from thinking them as secretive, expressive, magical or suspenseful. Each approach comes with its own benefits and concerns, but aims to create the best spectator experience. Around gaming, it has been reported that spectators most appreciate games designed to support visible actions and tactics, units in competitive play and emotions evoked during competition [7].

In addition to the multitude of technological problems related to wearable computing, social acceptability forms a noteworthy barrier. The bottom line being, as described by Rantanen et al [8], in addition to the technological features provided by a piece of clothing, it also has to provide the esthetical and functional properties one expects from clothing in general. Several issues related to wearable computing can be in opposition to these required properties, such as bulk [9] and dangling objects [10]. With wearable controllers even the placing of the controls has been reported to have an impact on the social acceptability. Holleis et al [10] found in their study that controllers on trousers, wristbands or separate bags received acceptance. On the contrary, the upper body, like a shirt or a scarf, was generally seen as not acceptable. Interestingly, Gabaret et al [11] found out that control has an effect on the social acceptability. The less control the wearer appeared to have over a wearable device, the more acceptable the device seemed to be to the ones around you. This notion was based on designing and comparing three different performative and artistic wearable computing devices.

The most interesting performative wearable devices in regards to our study are TQualizer [12] and BubbleBadge [13]. The T-Qualizer displays a music-related working equalizer on a t-shirt. The BubbleBadge is a wearable display designed to look like a brooch. As a brooch, it is meant to be placed on your chest, thus supporting face-to-face interaction.

\section{User Study}

To answer our research question, we planned a field trial with 10 participants. The age of the participants varied between 19-33, with 6 male and 4 female participants. Since we were interested in social acceptability issues that might arise if entertaining, wearable technology was introduced to workaday environment, we thought it best to 
actually bring the technology to users. Therefore the study was conducted at a university campus with both students (6) and staff (4). All participants were recruited on the spot. Each of the participants was asked to wear our Tic-Tac-Toe T-shirt for a period of approximately one hour. Participants were encouraged to go about their daily duties as usual, not to let this experiment be in the way of their normal routines and not to avoid any of the customary situations that might occur. The length of one hour was chosen based on the rhythm of life at the campus. Participants were recruited during their breaks and the test period ended during the expected next break. Thus, the hour long cycle consisted of going somewhere, staying somewhere and coming back from somewhere. These places, called somewhere in this case, were classrooms, auditoriums, office spaces, cafes and lunch restaurants. A normal day at the campus constitutes of many of these cycles, and extending the experiment would have only resulted in multiple similar cycles. Also, due to the design of our t-shirt, more prolonged experiments were expected to make the t-shirt mundane in a relatively short period.

To get an idea on what was going on while the participants wore the t-shirt, we applied a light version of ethnographic methodology based on a form of participant observation [14]. To his aim, 5/10 participants were shadowed. With shadowing the focus was on getting insight on the reactions of both the "performer" and the "spectator". In this case, the performer is the person wearing the t-shirt and the spectator anyone in the vicinity of the t-shirt. To get this insight, a moderator followed the user for the whole time the t-shirt was worn and took notes. Both the performer's impressions and comments and the observed reactions of spectators were documented. 5/10 users were shadowed. The rest were expected to spend most of their time in areas that we couldn't access due to drawing too much attention to ourselves. Dividing the users into two groups enabled us to evaluate, at least on a superficial level, if the shadowing had had any impact on the performers' answers to the questionnaire. Naturally, during the shadowing the moderator tried to be as inconspicuous as possible.

During shadowing, the comments of the users were written down as well as the reactions and the comments from spectators. Since the users went about their business as they usually would and the moderator aimed not to be intrusive, we weren't able to get all the comments made.

Participants weren't explicitly encouraged or told to draw attention to the shirt. In the short brief we gave about the study, we informed the participants that the t-shirt was "a display" for an actual ongoing tic-tac-toe game played on the Internet. The participant would hence basically act as a display for it. The participants were given a mobile phone to give a more authentic feel of an actual online game. We told the participants that the mobile phone was downloading the game from the Internet. We chose to fabricate the story of an actual online game to support the feeling of actually being interactive and as the T-shirt's game data in reality could benefit of being drawn from the Internet.

After returning the t-shirt to us, or when the shadowing moderator ended the session, the participants were asked to fill out a questionnaire. In a situation, where someone acted as a spectator for a longer period of time, the spectator was also asked to fill out a form that was especially drawn up for this type of a situation. The questionnaires consisted of both rating scales and open-ended questions about performer experience, spectator awareness, acceptability, interest towards the technology and areas of improvement. In addition, the participants gave rich verbal commentary after 
the test session. These comments gave us knowledge that weren't present in the filled out questionnaire. In total, the combination of shadowing, questionnaires and verbal commenting gave us a thorough understanding of the results.

\section{Results}

The participants wore the t-shirt in varied locations, thus meeting a varied amount of possible spectators and varied level of direct interaction with others. Based on our observations the t-shirt was worn in the corridors, small classrooms, a large auditorium, small office rooms, open office spaces with cubicles and in cafes. The most common reactions the users met with while wearing the shirt were looks, (hidden) stares, smiles, laughter, and "raised eyebrows". The users didn't find this attention in the least disturbing. These overall reactions evoked by the shirt were quite subtle in nature and according to the users even surprisingly so. A comment made by one of the participants serves as an example on this: "I was surprised, because it felt like people were paying less attention to me than usual." (User 7, Female) These notions by participants seem to suggest the presence of Goffman's [2] 'civil inattention', possibly even in an amplified form. It's socially acceptable to e.g. glance at a person you're passing by in a corridor. However, based on the observations, the glances by passers-by were very discrete and sometimes even missing altogether. Consequently, the observations made during shadowing support these partcipants' notions of somehow "being overlook".

The avoiding of eye contact, looking through someone etc. are typical forms of civil inattention. And this was the case in many situations. Our observations noted glances from a distance, but a lack of glances from shorter distances. It seemed that the spectators noticed the performer and that "something was up" from afar. So when they got closer, they were careful not to stare etc. In the context of civil inattention this could be interpreted as a sign of trying to assure that no embarrassment to one self or the performer would follow.

All the participants $(6 / 10)$ that showed the shirt to someone did so only to people they already knew. The most common reasons for showing the shirt to someone were: the users thought the shirt was fun and/or wanted to see the reactions of their friends/co-workers/fellow students. Also, by drawing attention to the shirt themselves, the users reported feeling more in control of the situation. The users, who didn't show the shirt to anyone explicitly, explained this being due to not seeing anyone they knew. Since the users were instructed to go about their business as usual, a few of them reported it being useless or meaningless e.g. "to sit in one's cubicle where there are no people around." (User 1, Female). This creates one limitation to the people's willingness to wear the shirt to places, where interaction around the shirt might not naturally occur. If there's no one to show the shirt to or no one to take a look at it, there seems to be little point in wearing it at all. This issue didn't come up with participants, who wore the shirt for their lunch or coffee breaks.

When compared to the findings with BubbleBadge [13], where the most fun was had by the spectators, it is interesting to realize the impact of civil inattention. It was clear from the users' responses that when attention was not received, the biggest factor for fun came from sharing. This sharing most arose in the form of showing the 
shirt to friends, who are natural recipients for sharing. Additionally, as no active or forthright attention or actions exhibited by strangers were reported, it would seem that the social interaction between the spectator and performer was greatly influenced by social closeness instead of just the actual setting. During shadowing it was also observed that social aspects of hierarchy might play a part in the matter. "Why did I turn on my heels? That's a good question, I didn't even think about it. Those are my bosses and if they saw with this shirt on I'm sure they'd start making jokes or something. " (User 7, Female)

When the shirt was shown to somebody, the spectators were mainly interested in finding out how the shirt works. As the shirt was designed to be somewhat magical, based on the taxonomy of Reeves et al. [6], this question was to be expected. When the social situation allowed the question to be asked, the spectators naturally wanted to understand "the magic". The spectators also asked what does it do and why the participant was wearing it. Two out of ten participants reported that someone had expressed interest in doing something with the shirt themselves - they either wanted to be able to play the game or wear the shirt. 8/10 participants wanted to be able to have some control over what's happening on and with the shirt. Most common concern was that the possible content of the shirt might be something the participant would find meaningless or being opposed to personal taste or values. "I'd like to decide what I'm displaying, yes. I wouldn't want to e.g. advertise McDonalds or a band I really don't like." (User 9, Male) Also the ability to turn the shirt on and off was mentioned. The tic-tac-toe game currently displayed on the shirt was seen as ok, but almost all of the users suspected that it wouldn't engage spectators for long. However, this wasn't seen as a big problem by the participants since the users didn't expect anyone to look at the shirt "for hours" anyway (User 10, female).

When choosing to utilize Tic-Tac-Toe in the design, we acknowledged that one design would not perfectly suit all participants. Naturally, when choosing a piece of clothing to wear, the clothing is often chosen based on the most appealing design. Our decision to use Tic-Tac-Toe was influenced by wanting an interactive design that still could be about self-expression and be appealing. Based on the feedback from the participants, the idea behind the idea was appealing, but not the current design of it. The participants stated that in an everyday situation the design should be more refined. When evaluating the current appeal, the shirt received an average of 2,4/5. On the other hand, the participants reported rather a high willingness (avg. 4,1/5) to wear the shirt again, based on how it suited their social surroundings. Some users even expressed enjoying the possibility "to be a little on view" (User 3, female) as a motivation for wearing the shirt. These results combined confirm similar findings made by Rantanen [8]. That is, in addition to the appealing technological features, wearable technology has to provide the esthetical and functional properties one expects of clothing in general.

Even though the average for the willingness to wear the shirt suggests that the everyday surroundings didn't present a problem for wearing the shirt, the answers to open-ended questions offer a corrective to this. The users listed suitable situations and places for using the shirt as follows: bar, party, for opening up a conversation, a night out, concert and nightclub. Work, business meeting and formal occasions were on the top of the list of the most inappropriate places to wear the shirt. On the other hand, for some participants the shirt "gave on excuse" to be on view. A few spectators also 
brought up this matter, combining it with what they termed to be the personality of the user. "It's ok I guess, but for X (User 6) to be wearing that... it's not really his style, his thing, if you know what I mean? It's not his personality."

When talking about the idea of the t-shirt, the participants also stated understanding that the idea of being a display on the move requires that the information presented has to be short. The users suggested that in addition to games (Worms, Tetris, Super Mario, Chess) the shirt could display e.g. weather forecasts, advertisements, news and videos. Using the internet for content retrieving was seen as a good idea and facilitating many varied options for the content displayed.

\section{Conclusions}

In this paper we have presented a T-shirt that portrays an on-going game of Tic-TacToe. With the help of the T-shirt, our goal was to understand the impact of civil inattention with wearable performative clothing. For this goal, we conducted a user study of 10 participants, who each wore the T-shirt for an hour in their everyday surroundings. To fully understand the different aspects of the matter, we dug deeper into the subjective cognizance of the participants with shadowing, questionnaires and discussions.

Our results indicate that civil inattention exists when using performative clothing. Our participants reported noticing some glances, but at the same time, noticing also an interesting lack of glances. Similarly our observations based on shadowing, support this notion. To compensate the lack of attention to something that the participants felt excited about, the participants chose to share this experience with their friends.

The participants also suggested acceptability towards utilizing wearable technology as a performative and eye-catching element. Our results were also in alignment with previous studies showing that wearable technologies need to provide aesthetic and functional properties, just like clothing in general. Based on our relatively small sample data, we cannot make deeper claims of the reception of such technologies, but we feel that this is a good starting point for further investigation related to social acceptability. And even the fact of agreeing to wear the shirt in the populated surroundings, where the users inhabit and work, can be considered to be at least a weak sign of acceptance for introducing wearable, personal technology for such surroundings. Since the study was the first done with our T-shirt, it acts primarily as an explorative study for testing and mapping out the social acceptability issues. The results highlight the acceptability issues that arise when introducing a new technology into the mix. To this end, the study setup provided excellent results. On the other hand, the results are mainly relevant to the introduction, not long-term use. To understand the long-term issues, more research needs to be done.

For future work, we aim to support and study features for richer interaction and enhanced spectator experience.

\section{References}

[1] Rico, J., Jacucci, G., Reeves, S., Koefoed Hansen, L., Brewster, S.: Designing for Performative Interactions in Public Spaces. In: Proc. Ubicomp 2010, Copenhagen, Denmark (September 23-29, 2010) 
[2] Goffman, E.: The Presentation of Self in Everyday Life. Doubleday, Garden City (1959)

[3] Gajadhar, B.J., de Kort, Y.A.W., Ijsselsteijn, W.A.: Shared funn is doubled fun: Player enjoyment as a function of social setting. In: Markopolous, P., de Ruyter, B., Ijsselsteijn, W., Rowland, D. (eds.) Fun and Games 2008. LNCS, vol. 5294, pp. 106-117. Springer, Heidelberg (2008)

[4] Gajadhar, B.J., de Kort, Y.A.W., Ijsselsteijn, W.A.: Rules of Engagement: Influence of Co-Player Presence on Player Involvement in Digital Games. International Journal of Gaming and Computer-Mediated Simulations (2008) (in press)

[5] Jonsson, F.: A Public Space of their own. A Fieldstudy of a Game Café as a Third Place. In: Nordic DiGR, Stockholm, Sweden (August 16-17, 2010)

[6] Reeves, S., Benford, S., O'Malley, C., Fraser, M.: Designing the spectator experience. In: Proceedings of CHI 2005. ACM, New York (2005)

[7] Cheung, G., Huang, J.: Starcraft from the Stands. In: CHI 2011, Vancouver, Canada (May 7-12, 2011)

[8] Rantanen, J., Karinsalo, T., Mäkinen, M., Talvenmaa, P., Tasanen, M., Vanhala, J., Alfthan, N., Impiö, J., Malmivaara, M., Matala, R., Reho, A.: Smart Clothing for the Arctic Environment. In: Proc. ISWC 2000, p.15 (2000)

[9] Dunne, L.E., Ashdown, S.P., McDonald, E.: 'Smart Systems': Wearable Integration of Intelligent Technology. In: International Center for Excellence in Wearable Computing and Smart Fashion Products, Cottbus, Germany (December 9-11, 2002)

[10] Holleis, P., Schmidt, A., Paasovaara, S., Puikkonen, A., Häkkilä, J.: Evaluating capacitive touch input on clothes. In: ter Hofte, G.H., Mulder, I., de Ruyter, B.E.R. (eds.) ACM International Conference Proceeding Series, pp. 81-90. ACM, New York (2008)

[11] Garabet, A., Mann, S., Fung, J.: Exploring Design through Wearable Computing Art(ifacts). In: Extended Abstracts CHI 2002, Minneapolis, Minnesota, pp. 634-635. ACM Press, New York (2002)

[12] T-Qualizer, http: / / www.tqualizer.com/

[13] Falk, J., Bjork, S.: The BubbleBadge: A Wearable Public Display. In: Proceedings of CHI 1999, pp. 318-319 (1999)

[14] Chicago school (sociology) (April 7, 2011), http://en.wikipedia.org/wiki/Chicago_school_(sociology) 